\title{
Modeling Content and Expression of Learning Objects in Multimodal Learning Management Systems
}

\author{
Giorgio Brajnik \\ Dip. di Matematica e Informatica, \\ University of Udine, Italy \\ giorgio@dimi.uniud.it
}

\begin{abstract}
The paper discusses the relation between accessibility and multimodality of learning objects. I present a framework, rooted in linguistics, that supports a clear distinction between content and expression of learning objects. The framework supports different types of content elements, and different expression elements in which content can be rendered, which include different expression media, multimedia components and spatial/temporal/hyperrelationships. I show what accessibility means in such a framework, and what the consequences of accessibility are in terms of automatic transformations of expression that can occur on student platforms. I argue that accessibility of learning objects, though making them multimodal, is not a satisfactory solution from the viewpoint of learning effectiveness. I claim that content modeling needs to be a crucial step in the development of any learning object, and propose notations based on conceptual maps and UML diagrams.
\end{abstract}

Keywords: knowledge modeling, accessibility, operability of user interfaces.

\section{Introduction}

One function of Learning Management Systems (LMS) is to deliver one or more learning objects (LO) to students that access the LMS from various user platforms and in different situations. A student might access the LMS at home through a PC with a graphical browser; another from her PDA while sitting in a train; a third one using a PC at the university library, with audio turned off; a blind student might use a screen reader in a classroom during a lecture; a fifth one might have selected a user profile so that podcasting is her preferred way to access the instructional material; and a sixth one usually prefers to print lessons on a low-resolution and slow ink-jet color printer.

Other functions of LMS are to permit students to solve exercises and submit projects, to access collaborative features of the LMS (like a chatroom, a forum, a blog to view others' postings or to contribute with new content, to get in touch with other students, tutors, instructors), to access help/support sections of the LMS, or finally to use administrative services (to enroll to a course, to withdraw from a joint project, to view one's own ratings, etc.). In all these cases, students may access the LMS with a variety of devices, in a variety of situations.

In all these cases designers (of the LMS and especially authors of LOs) have no control on the platform adopted by the student to use the learning material. Yet, in all 
these cases students should experience high levels of quality, especially in terms of usability, accessibility, engagement, memorability, and learnability [7]. In fact, learning effectiveness of LOs is heavily affected by motivational and persuasive factors embedded within LOs. Also the LMS may affect motivation, persuasiveness and learning effectiveness: usability mishaps, accessibility barriers, media interference within LOs and/or LMS may dramatically reduce teaching effectiveness. Furthermore, not every media is equivalent to the other ones with respect to learning effectiveness: one could conceive using podcasting for an introductory lecture on operating systems, but wouldn't use it for a lecture on Cauchy's calculus theorem.

Very often multiple media are used to enhance user experience, sometimes in a redundant way (like adding sound effects associated to user inputs), sometimes to complement each other (like using a visual simulation of electrical current in addition to a textual description of the phenomenon being simulated), sometimes as alternatives (like adding textual captions to a video for the benefit of those who cannot see the images).

What is the extent to which LOs can be adapted (or made adaptable) to these different learning situations? What kind of guidelines need to be given to LOs authors in order for them to provide redundant information? Is accessibility enough?

In this paper I explore these questions, laying a foundation upon which to look to issues like accessibility, multimedia, multimodality of user interfaces and LOs. An important conclusion is that content, defined in a sounder way than what is current understanding for websites and applications, has to be modeled separately from expression. I propose practical ways to isolate and model it.

\section{Content Versus Expression}

It is important to be able to separate content from expression within a LO (actually, the same applies also to the LMS hosting the LOs) in order to be able to clearly distinguish two important types of questions: those dealing with the meaning that has to be conveyed by the LO, and those dealing with how meaning is to be expressed. If a clear separation can be achieved, then it would simplify decisions regarding what to say, which arguments to draw upon in order to be convincing, which level of detail should the LO reach, what kind of external references to include, etc. It would simplify also decisions on the different ways in which the same meaning can be expressed, which media and combination of media would be more effective, and how the LO would be used (using different media and different modalities).

We base our distinction between content and expression on a linguistic approach, and assume that a LO can be thought of as a communication artifact (a message), involved in communication processes that occur between the LOs' authors and students. Additional details are available on [2]. Hjelmslev [6] suggested to consider a message as the combination of content, which corresponds to the meaning being conveyed by the message, and expression, which corresponds to the ways in which the meaning is expressed so that it can be perceived by human beings. Both content and expression can be discussed in terms of substance and form. 


\subsection{Content of a Learning Object}

The content of a message is the result of a process of sensation-perceptioninterpretation guided by the context of the addressee (in the case of a LO the addressee is the student). By context I mean a combination of the physical situation the user/student is in (physical environment, input/output interaction devices, posture, light and noise conditions), his/her goals (final/behavioral/life-time goals [5]), beliefs, attitude and motivations.

Substance of content, for a generic LO, can be classified in the following types.

- Information of interest, which corresponds to the primary information that is being conveyed by the LO, and is what a student would expect to see/read/hear/learn to satisfy his/her final goals. Concepts belonging to the domain of interest, explanations, questions, answers, clarifications, examples, counter-examples, descriptions of phenomena are all information of interest. Also help material, tooltips and usage instructions belong to this category, as well as (external or internal) promotional messages.

- Bearing information, which supports the user/student in navigating and interacting with the system. Bearings can be further classified as:

- Location information, that provides clues regarding the current position in the virtual space implemented by the LOs and the LMS. Examples are breadcrumbs [7], sentences like "question 3 our of 6", the lecture or page title, the title of an inset in the page, the label "on" attached to an animated switch which is part of a simulated electric circuit in the LO.

- Direction information, which describes possible destinations that can be reached, steps that can be performed or activities that can be started. Examples include labels of links/buttons for moving among pages, menu entries, navigation bar entries, labels of buttons to control a simulation.

- Access information, used to activate functionalities of the system and achieve some response. To enable choice, very often access information is coupled to direction information. Access information can be split in:

- Sequential access schema, that support a linear scanning of content units that are sorted according to some pre-defined criterion. Typical examples are the previous/next pairs of buttons that support scanning of pages of a tutorial.

- Indexed access schema, that support access to units in any order. Examples include navigation bars, list of links, single buttons/links, drop-down menus.

- Filtered access schema, that support operations for restricting the set of possible destinations. The most typical example is in a faceted classification interface [7]; other examples are forms used to select lectures, exercises, or postings in a forum.

- Functional information, that has to be provided by students in order to complete certain tasks. This includes, for example, answers to exercises.

Form of content refers to possible relationships that can be defined among content units. These relationships usually derive from a taxonomy or ontology of the domain: when certain classification criteria are used (f.e. by subject, by audience, by function, 
etc.)[10], appropriate categories are identified and proper relationships among them are defined (f.e. "type-of", "instance-of", "cause-of", etc.). Other relationships can be identified by adopting the Rhetorical Structure Theory (initially applied only to text [9], but more recently also to multimedia objects [3]). This theory can be used to determine if content units are consistent by identifying patterns of relationships that should hold among content units (f.e. unit A contrasts unit B, A is an example of $\mathrm{B}, \mathrm{A}$ provides details for $\mathrm{B}$, A explains $\mathrm{B}$, etc.). Finally, content form includes also abstract task models, specifying combinations of steps (like sequences, loops, conditionals, fork/join points) that need to be followed in order to achieve the task goal.

Consider the example shown in Figure 1. From the content perspective (i.e. by not considering expressive aspects), its substance includes several location information providing an overview of the page (f.e. "The Magnetic Circuit", "Magnetic Force"), location information (f.e. the knob position set to $5 \mathrm{v}$, the display on the switch unit displaying " $5 \mathrm{~V}$ ", etc.). Except for the knobs that can be turned, this page does not include any direction information (buttons on the bottom of the page, labeled with "back" and "next", do not describe any destination; they would if they were labeled with the name of the destination, like "magnetic flux" rather than "next"). The content includes also concepts like magnetic flux, voltage, currents, magnetomotive force, switch units, voltage and current measuring units, current measuring units, lamps, electrical circuits, series connection, etc.; these constitute the information of interest.

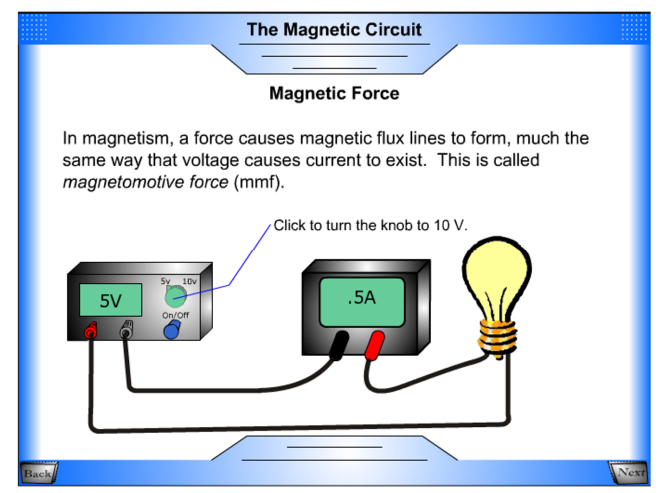

Fig. 1. A screenshot of a scene of the LO on magnetic circuits developed by T. Bartelt and J. Janikowsi, 2006 and published by Wisc-Online, www.wisc-online.com/objects/index_tj.asp ?objID=DCE14906

The form of the content of this example includes several relationships. Some are expressed in the text introducing the concept of magnetomotive force: we can see that authors adopted the magnetism-as-electricity metaphor, which is a relationship. Other relationships concern the electromagnetic phenomena being shown by the simulated circuit (f.e. when the button is set to "on" the lamp turns on; when the voltage knob is set to $10 \mathrm{v}$, the lamp gets brighter). While the top part of the screenshot introduces the metaphor, the bottom part does not do that: it simply allows the student to refresh his/her (assumed) knowledge of electrical circuits, without reinforcing the metaphor and without providing any support for understanding magnetomotive force, other than 
refreshing the analogy. The content of that page is also part of the abstract task of studying each unit of the LO; such a task prescribes to sequentially go through all the units, in the prescribed order. The order of the units is dictated by prerequisite constraints among concepts and the adopted teaching strategy.

\subsection{Expression of a Learning Object}

Expression of a message deals with the ways in which content can be perceived by humans. Therefore (except for brain-computer interfaces) expression requires that some physical medium is used to represent physical differences that we can sense (pressure waves in air, ink areas on paper absorbing light, pixels emitting light, etc.). While the physical medium depends on the interaction device being used, at a more abstract level we can identify expression media, which are usually classified into text, image, sound, or video. Components expressed in any of these media through specification of associated style attributes (f.e. font faces, colors, sizes, textures, voices, tones, frame-rate, etc.) are the building blocks of the expression of a LO, and constitute the substance of the expression. Appropriate media and style attributes are also used to represent controls upon which the student can act, f.e. the particular look and feel style used for representing the button to move to the next unit.

The form of expression involves space and time. Components (each with its own expression medium) can be combined together in a large variety of ways by choosing appropriate spatial, temporal, spatio-temporal or hyper structures. Text and images are static components that occupy some area in the bidimensional space provided by the browser window; their relative position is an expressive relationship. In other cases, text and images may be part of a dynamic medium (video): in the simulation that the LO of Figure 1 offers, the space occupied by images does not change over time, but other components and attributes do: text labels change between " $5 \mathrm{~V}$ " to " $10 \mathrm{~V}$ ", the picture of the knob changes when it is "turned", the color of the lamp changes. In other LOs there might be sounds as well, with their own evolution over time, often synchronized with text, images or user actions.

The form of the expression is the result of choices that the LO authors made in order to achieve certain pragmatic effects on students; f.e. if an image is below some text, then it is either semantically nested or it follows the text. In both cases the student is expected to read the text first, which is more important.

In addition to temporal and spatial structures, within a LO there is also a hyper structure. Over time, depending on user-initiated events, the interface "moves" across this hyper structure. In general, the hyper structure reflects some of the relationships that were identified in the content and implements appropriate access and bearing information (f.e. navigation bars, drop-down menus, pop-up menus and tabs are four possible different expressions of the same combination of destination, location and access information). In our example, the hyper structure implements the linear structure prescribed by the task model, and is a sequence of units. Within a unit, several possibilities exist. For example, in the unit shown in Figure 1, the student can repeatedly run and re-run the simulation by switching the voltage knob; but the student cannot turn the current off once the circuit is on, unless the unit is restarted (by returning to the previous one and then moving on to the next). To run the simulation for the first time, the student has to click on the central area of the "on/off" 
push-button; subsequently the only available action is clicking on the central area of the voltage knob. Consider also that the hyper structure may be time-dependent: f.e. until the student runs a simulation of the circuit there is no way to move forward to the next unit.

While in this discussion I focused primarily on a single LO, the same kind of properties apply to the LMS. Navigation bars, menus to invoke functionalities that are unrelated to the LO are all examples of other expression components that implement content that is part of the LMS.

LOs exploit multimedia (which is meant as integration of different media so that their combination achieves effects that are more than just the sum of the individual components) in order to obtain pragmatic effects on students, like enhancing attractiveness, achieving persuasion, capturing and retaining attention, increasing usability, enhancing memorability (f.e. it is known that images are more memorable than text, which is more memorable than spoken words, and that static images are more memorable than videos). However, unwanted interactions between media can produce also interference which prevents proper interpretation (f.e. when changes of text size rearrange the components in the page, perhaps removing some of the visual clues that are needed to interpret the page).

Multi-modality is not directly bound to the expression being used in the LO, as it depends on the kind of physical actions that students can execute (on appropriate physical interaction devices like keys, switches, joystick, knobs, wheels, cameras, etc.) in order to carry out common operations (like activating a button, selecting an option, dragging and dropping something, etc.).

\section{Accessible Learning Objects}

With "accessible LO" I mean a LO that is as effective for disabled students as it is for non-disabled ones [11], where "effective" is meant in terms of task accuracy and completeness and not in terms of teaching effectiveness. "Disabled student" include the typical categories of disabled users of the Web, namely: people with no vision using screen/Braille readers and keyboard; low-vision users of screen magnifiers; deaf users using sign language or non-audio information; motor disabled people using only the keyboard, special keyboards or mice, scanning devices, body gestures, or speech recognition; cognitively disabled people that may use speech recognition, word predictors when typing and may need to use simple language and additional icons.

Accessibility clearly depends on the user platform, which includes the operating system, its user interface components (f.e. KDE), the underlying accessibility architecture (f.e. MSAA), and the assistive technology (f.e. the screen reader, the screen magnifier, a modified keyboard, an on-screen keyboard, etc.).

Provided that the LMS is accessible, and that the user platform includes assistive technology required by the student, the expression of the LO depends on the user platform and on specific options set by the student during usage of the LO. For example, text could be read aloud; images could be "transformed" into spoken words; scenes of a video could be enriched with textual captions describing them; audio content could be transformed into textual transcripts. These changes in expression involve inter-media transformations (f.e. text to spoken words), intra-media 
transformations (f.e. by changing the geometric properties of space when using a screen magnifier to enlarge the screen, or when changing the text size), temporal transformations through new synchronization of events (f.e. by using audio signals to notify a user of a screen reader that a certain feedback message has appeared in a location that differs from the current focus of interaction) or slow-down of an animation/simulation and de-contextualization of information (f.e. when the user of a screen reader extracts and lists all the links in a page, so that each link is rendered out of its original context).

Some of these transformations affect interaction modality. For example, in an accessible LO new operations may be made available, like the ability to extract and scan links in a page, or page headings, or the ability to jump directly to the content of the page, or the ability to move back and forth through items of a list. Since interaction devices used by students are not constrained by the LO author, to carry out these operations students may use any devices: only the keyboard, only a sip-and-puff sensor, a virtual keyboard on the screen, voice commands, etc. Obviously, not every combination of expression and interaction devices makes sense: f.e. blind users of a screen reader don't usually use mice because they do not provide sensible feedback.

Provided that a LO is accessible, a first necessary condition is that its content is available to wide range of students using several platforms. The LO can support transformations of its expression so that meaning is not lost (i.e. neither interest nor bearing information is lost). A second necessary condition is operability of the LO so that each relevant operation (needed to supply functional information or to operate on access information) can be performed through appropriate actions (i.e. operations specified in the task model part of the content are not lost). We call these conditions invariance of content with respect to changes in expression and interaction modalities.

\subsection{Agents Affecting These Transformations}

In order for these transformations to occur so that invariance of content holds, a number of enabling conditions need to be met.

1) The user platform should support all required transformations, through standard assistive technology, browsers, plug-ins and accessibility architectures.

2) The technologies used by the LMS and the LOs (f.e. HTML, CSS, JavaScript, SMIL, Flash, PDF) should be supported by the user platform, and the technologies themselves should support the required transformations.

3) Authors of LOs need to provide the required redundant expression in the different media that can be used by students (f.e. textual descriptions of video scenes) and required specifications to support transformation of expression (f.e. synchronization constraints so that captions are rendered at the right time).

Thus, from the perspective of the LOs authors, accessibility requires them to clearly identify all the content units (the substance and form of the content, see Section 2.1) and make sure that 1) interest and bearing information can be transformed into all possible media that might be available in students' platforms, 2) that the transformation are complete (f.e. all bearing information is transformed) and 3) that operability is guaranteed (f.e. all functional information and access information can be operated upon by students). 


\subsection{Modeling Content}

One way to achieve invariance of content is to make sure that authors of LOs can actually think about content of LOs independently from their possible expressions. Appropriate notations can support such a process: Figures 2 and 3 show examples of content models expressed through conceptual maps, and through UML activity diagrams. Conceptual maps (Figure 2) can describe a taxonomy/ontology and highlight relevant concepts and their relationships. In this way, authors can lay down the domain of interest to be covered and decide which concepts will be dealt-with by the LO.

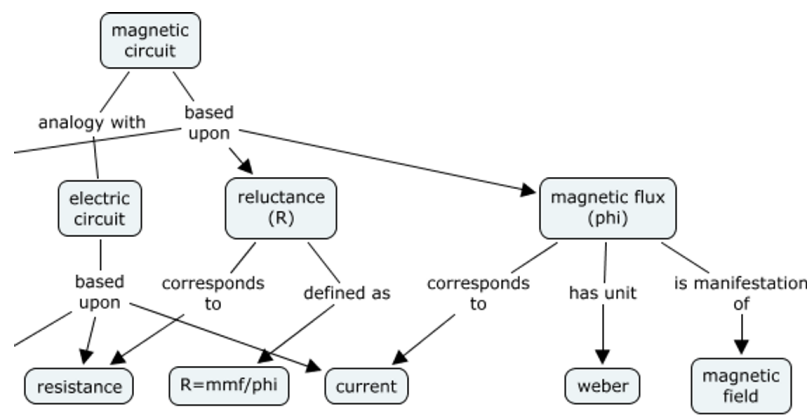

Fig. 2. Part of the basic domain concepts covered by the LO shown in Figure 1

Finally, Figure 3 shows part of an activity diagram [4] that specifies the activities that the LO does, interlaced with the activities that students are expected to perform. For example, the LO begins by presenting an information of interest stating the analogy between magnetic and electrical circuits. The task model specified by the diagram requires the student to act in order to move to the next unit, entitled with the location information "Magnetic force", and containing as information of interest an introduction to the concepts of electric force, voltage and current. A further student action leads to the description of the phenomena regarding the basic electrical variables, specified by the UML object called "electric circuit", another information of interest which, in the expression shown in Figure 1, is implemented through the simulation involving the images. The student can explore different scenarios (using the indexed access information that supports setting the voltage to 0,5 or $10 \mathrm{v}$ ) regarding these phenomena until s/he continues to the next unit.

An activity diagram is useful because it can highlight, as UML objects, the specific contents that the LO should present, synchronized with the activities these objects are associated with. For example, the object "electric circuit" is shown when the unit entitled "Magnetic force" is shown, but also when the students "activates" the subsequent system activity called "present details of elec. circ.". Such an object disappears afterwards.

What these diagrams represent is totally independent from expression. It could be implemented with the expressions shown in Figure 1 (namely text that changes, animated images, specific buttons, specific screens), or in a totally different way (like a voice narration of the electrical phenomena captured by object "Electric circuit"). 


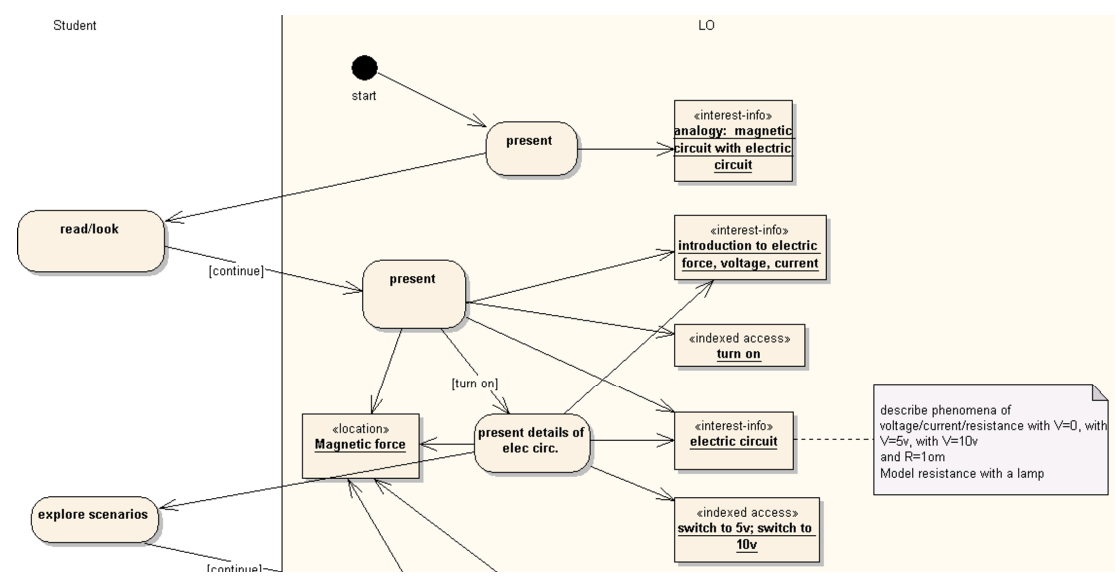

Fig. 3. Part of the activity diagram describing actions of the student and of the LO shown in Figure 1

The same freedom is available for implementing how the student is expected to move back and forth between units or how the different scenarios $(0,5$ or $10 \mathrm{v})$ are to be selected.

\section{Discussion and Conclusions}

Accessibility is a starting point for multimodal LOs, but unfortunately it is not enough. In fact, while accessibility guarantees levels of task effectiveness, specific expression choices made by the designer contribute to the learning effectiveness. As we've already seen, different media lead to different memorability degrees; interpretation of images requires less effort and time than text; spoken words might not be understood if there is a long interference with other sounds; static and moving images can be used to attract attention, they can provide affective clues, they can compensate text; sound icons can attract attention. Videos and animations can attract and hold students attention, but dominate on other media that are rendered at the same time. With proper use of expression, authors of LOs can implement contrast between components and can assign different relative importance to them by using emphasis. Unfortunately, automatic transformations of expression are not guarantee to preserve these properties.

As a consequence, content invariance does not guarantee the same level of learning effectiveness. In my view the only solution for having adaptable multimodal multimedia LOs (i.e. such that their expression change according to the use situation but without affecting the learning effectiveness) is to explicitly design different expressions, each geared towards a specific use situation [2]. Therefore, rather than relying on automatic adaptation of expression achieved through the transformations we discussed above, the author has to provide them explicitly. But also in such a case, content modeling is crucial, because authors can focus first on content and then, independently, design all the alternative expressions. 
I believe that modeling content (separately from its possible expressions) is a fundamental step for enabling truly accessible and multimodal LOs. Appropriate content modeling notations and systems need to be adopted by designers, but fortunately they are already available. By separating content from expression, one can more easily determine which content units should be made available to students, how they could be made available to students, and how students might operate on them.

\section{References}

1. Brajnik, G., Toppano, E.: Creare Siti Web Multimediali, Addison-Wesley/Pearson Education Italy (2007)

2. Brajnik, G.: Achieving Universal Web Access through Specialized User Interfaces. In: Stary, C., Stephanidis, C. (eds.) User-Centered Interaction Paradigms for Universal Access in the Information Society. LNCS, vol. 3196, Springer, Heidelberg (2004)

3. Bocconi, S., Nack, F.M., Hardman, L.: Using rhetorical annotations to generating video documentaries. Technical report CWI INS E0501 (2005)

4. Conallen, J.: Building Web Applications with UML. Addison-Wesley, Reading, MA (2003)

5. Cooper, A., Reimann, R.: About Face 2.0: the Essentials of Interaction Design, Wiley and Sons (2003)

6. Hjemlslev, L.: Prolegomena to a Theory of Language, University of Wisconsin Press (1961)

7. Holzinger, A.: Fundamentals of Human-Computer Interaction (HCI) for e-Learning. In: Mittermeir, R.T. (ed.) Innovative Concepts for Teaching Informatics. Vienna (Austria): Ueberreuter C, pp. 157-159 (2005)

8. Instone, K.: Location, Path \& Attribute Breadcrumbs (visited October 2006) http://www.user-experience.org/uefiles/breadcrumbs

9. Mann, W., Thompson, S.: Rhetorical Structure Theory: Towards a Functional Theory of Text Organization, Text, vol. 8(3) (1988)

10. Rosenfeld, L., Morville, P.: Information architecture, O’Reilly (2003)

11. Slatin, J., Rush, S.: Maximum accessibility: Making your Web Site More Usable for Everyone. Addison-Wesley, Reading, MA (2003) 